\title{
PredicCión DE EMISIONES DE CO Y HC EN MOTORES OTTO MEDIANTE REDES NEURONALES
}

\section{Prediction of CO AND HC EMISSIONS IN OTTO MOTORS THROUGH NEURAL NETWORKS}

\author{
Rogelio Santiago León Japa ${ }^{1}$, José Luis Maldonado Ortega ${ }^{1}$, \\ Rafael Wilmer Contreras Urgilés ${ }^{1, *}$
}

\section{Resumen}

En el presente trabajo se explica la aplicación de RNA (redes neuronales artificiales) para la predicción de emisiones contaminantes generadas por fallas mecánicas en motores de encendido provocado, de la cual se puede cuantificar el porcentaje de $\mathrm{CO}$ (\% monóxido de carbono) y el particulado por millón HC (ppm hidrocarburos sin quemar), a través del estudio de la fase de admisión del ciclo Otto, la cual es registrada por medio de la implementación física de un sensor MAP (Manifold Absolute Pressure). Se aplica un riguroso protocolo de muestreo y consecuente análisis estadístico. La selección y reducción de atributos de la señal del sensor MAP se realiza en función del mayor aporte de información y diferencia significativa con la aplicación de tres métodos estadísticos (ANOVA, matriz de correlación y Random Forest), de la cual se obtiene una base de datos que permite el entrenamiento de dos redes neuronales feed-forward backpropagation, con las cuales se obtiene un error de clasificación de $5.4061 \mathrm{e}^{-9}$ y de $9.7587 \mathrm{e}^{-5}$ para la red neuronal de $\mathrm{CO}$ y $\mathrm{HC}$ respectivamente.

Palabras clave: predicción, emisiones contaminantes, monóxido de carbono ( $\mathrm{CO}$ ), hidrocarburos no combustionados (HC), diagnóstico, redes neuronales artificiales.

\section{Abstract}

This paper explains the application of RNA (artificial neural networks) for the prediction of pollutant emissions generated by mechanical failures in ignition engines, from which the percentage of $\mathrm{CO}$ (\% carbon monoxide) and the particulate can be quantified. per million HC (ppm unburned hydrocarbons), through the study of the Otto cycle admission phase, which is recorded through the physical implementation of a MAP sensor (Manifold Absolute Pressure). A rigorous sampling protocol and consequent statistical analysis is applied. The selection and reduction of attributes of the MAP sensor signal is made based on the greater contribution of information and significant difference with the application of three statistical methods (ANOVA, correlation matrix and Random Forest), from which a base of data that allows the training of two neural networks feed-forward backpropagation, with which we obtain a classification error of $5.4061 \mathrm{e}^{-09}$ and $9.7587 \mathrm{e}^{-05}$ for the neural network of $\mathrm{CO}$ and $\mathrm{HC}$ respectively.

Keywords: prediction, pollutant emissions, carbon monoxide $(\mathrm{CO})$, non-combustion hydrocarbons $(\mathrm{HC})$, diagnostics, neural networks.

\footnotetext{
$\overline{1, *}$ Grupo de Investigación de Ingeniería del Transporte (GIIT), Carrera de Ingeniería Mecánica Automotriz, Universidad Politécnica Salesiana, Cuenca-Ecuador. Autor para correspondencia rcontreras@ups.edu.ec

(D) http://orcid.org/0000-0003-2142-3769, (D) http://orcid.org/0000-0002-3846-2599,

(D) http://orcid.org/0000-0003-2300-9457
}

Recibido: 15-05-2019, aprobado tras revisión: 05-11-2019

Forma sugerida de citación: León Japa, R. S.; Maldonado Ortega, J. L. y Contreras Urgilés, W. R. (2020). «Predicción de emisiones de $\mathrm{CO}$ y $\mathrm{HC}$ en motores Otto mediante redes neuronales». InGEniUs. N. ${ }^{\circ}$ 23, (enero-junio). pp. 30-39. DOI: https://doi.org/10.17163/ings.n23.2020.03. 


\section{Introducción}

En la actualidad, el área del transporte automotor representa una de las fuentes principales de contaminación atmosférica; en efecto la descarga de contaminantes al ambiente tiene su origen en el acelerado crecimiento poblacional y desarrollo de diversos centros urbanos, por lo que el deterioro de la calidad del aire es por fuentes: móviles (vehículos), fijas (industria) y de área (actividades domésticas y servicios).

El área vehicular (vehículos a gasolina y diésel) es uno de los principales emisores de combustibles fósiles quemados al medio ambiente, debido a los gases contaminantes que se producen durante el funcionamiento del transporte automotor, siendo las principales emisiones: monóxido de carbono $(\mathrm{CO})$, dióxido de carbono $\left(\mathrm{CO}_{2}\right)$, hidrocarburos no combustionados $(\mathrm{HC})$ y óxidos de nitrógeno $\left(\mathrm{NO}_{x}\right)$, de tal manera que afectan a la salud pública y equilibrio de los diferentes ecosistemas.

Por lo antes expuesto, es necesario desarrollar nuevas técnicas especializadas y metodológicas para conseguir diagnósticos asertivos de fallos mecánicos, como también de emisiones de gases de escape; simultáneamente el uso de redes neuronales artificiales y matemática computacional, a causa de la complejidad de análisis e interpretación de los parámetros de operación del MEP, de tal manera que se determinen los fallos mecánicos y las emisiones que estos provocan, en tiempos cortos de diagnóstico con optimización de recursos. La contaminación atmosférica tiene efectos nocivos para la salud de todos, así lo demuestra la investigación llevada a cabo por Ballester [1], quien muestra que en Francia, Suiza y Austria el $6 \%$ de la mortalidad y un número importante de nuevos casos de enfermedades respiratorias en estos países puede atribuirse a la contaminación atmosférica, siendo la mitad de este impacto debido a la contaminación emitida por los vehículos de motor.

La investigación de Restrepo et al. [2] estima alarmantes aportes de emisiones contaminantes que genera esmog y contribuye al efecto invernadero en la ciudad de Pereira. El estudio indica el aporte de contaminación de cada categoría vehicular según un software del modelo internacional de emisiones y una extrapolación, los resultados indican que vehículos particulares aportan más del $80 \%$ de las emisiones de $\mathrm{CO}$, el $60 \%$ de $\mathrm{CO}_{2}, 65 \%$ de $\mathrm{NO}_{x}, 40$ de $\mathrm{SO}_{x}$ y en las motos que aportan alrededor del $65 \%$ de PM (material particulado).

La utilización de redes neuronales es considerada una técnica de gran contribución en el análisis de parámetros internos de los motores MEP, así lo demuestra Li et al. [3] mediante la aplicación de una red neuronal para la predicción de las emisiones de $\mathrm{NO}_{x}$, el estudio utiliza relaciones de intensidad de radicales de llama, junto con la temperatura de llama y las emisiones de $\mathrm{NO}_{x}$, para entrenar la red neuronal.
Cortina [4] propone un modelo de predicción de concentración de los contaminantes en la ciudad de Salamanca (México), los contaminantes más críticos son $\mathrm{SO}_{2}$ y $\mathrm{PM}_{10}$, el modelo hace uso de redes neuronales artificiales (RNA) combinadas con algoritmos de agrupamiento, el estudio usa determinadas variables meteorológicas como factores que influyen en la concentración de contaminantes.

Es pertinente reducir las emisiones de $\mathrm{CO}, \mathrm{HC}$ y $\mathrm{NO}_{x}$ de los motores de combustión interna de encendido por ignición, porque son causantes de diversos problemas ambientales, como la contaminación del aire y el calentamiento global, Martínez et al. [5] utilizaron redes neuronales artificiales (ANN) para predecir las emisiones de escape de un MEP de 1.6 l, con el objetivo de optimizar el MEP, al reducir las emisiones de $\mathrm{CO}, \mathrm{HC}$ y $\mathrm{NO}_{x}$, cuyas entradas provinieron de seis parámetros operativos del motor y las salidas fueron tres emisiones de escape resultantes.

De igual forma, Fontes et al. [6] aplican redes neuronales mediante un perceptrón multicapa (MLP) con una capa oculta como un clasificador del impacto de la calidad del aire en la salud humana, utilizando solo datos de tráfico y meteorológicos como entradas. Estrategias paralelas y combinadas se pueden emplear para la determinación de concentraciones de emisiones, por ejemplo, un aprendizaje híbrido de la red neuronal artificial (ANN) con el algoritmo genético de clasificación no dominada - II (NSGAII) para mejorar la precisión y predecir las emisiones de escape de un motor a gasolina de encendido por ignición de 4 tiempos [7].

Se puede aplicar diferentes métodos para analizar y predecir emisiones, como el modelo de regresión lineal multivariante para analizar la relación entre contaminantes atmosféricos y factores meteorológicos. López y Pacheco [8] muestran que el benceno tiene como fuentes humo del tabaco, gasolineras, emisiones industriales y tubo de escape de automóviles en la zona urbana de la ciudad de Cuenca (Ecuador), genera un aumento en el número de casos clínicos tales como: asma $(36,34 \%)$, bronconeumonía (12,19\%), bronquiolitis (16,89\%), bronquitis $(6,29 \%)$, faringitis $(12,41 \%)$, neumonía $(11,73 \%)$ y rinitis $(3,67 \%)$, todo esto por el incremento en una unidad de su concentración; por otra parte, el $\mathrm{PM}_{10}$ presenta una relación positiva con la trombosis venosa ocasionando un incremento de 3,56\% de casos clínicos por cada unidad que incremente su concentración. Guadalupe [9] aplica una nueva metodología de modelación de emisiones contaminantes de fuentes móviles terrestres en Quito (Ecuador), el modelo internacional de emisiones vehiculares (IVE Model), el cual engloba una metodología tipo bottom - up, que reúne gran cantidad de información para conformar el inventario de emisiones.

Las estrategias de predicción de concentraciones de gases son variadas en la actualidad. León y Piña [10] 
presentan un modelo de predicción de emisiones $\left(\mathrm{NO}_{x}\right.$, $\mathrm{CO}, \mathrm{CO}_{2}, \mathrm{HC}$ y $\mathrm{O}_{2}$ ) aplicado en vehículos a gasolina, con la utilización de redes neuronales (ANN), las variables de entrada en la red neuronal son PME (presión media efectiva), rpm, carga y MAP, también el modelo predice la carga del motor, asimismo Contreras et al. [11] han propuesto un sistema de diagnóstico que puede detectar fallos mecánicos en motores de encendido por ignición de ciclo Otto, mediante redes neuronales (RNA), el sistema se sustenta en la utilización de las señales de los sensores MAP y CMP, y tiene un error de clasificación de $1.89 \mathrm{e}^{-11}$.

El sistema de predicción propuesto puede determinar emisiones contaminantes y los fallos mecánicos que provocan las determinadas emisiones, tales como: monóxido de carbono (CO) e hidrocarburos sin quemar (HC), el diagnóstico que realiza el sistema de predicción no lo realiza la ECU (Engine Control Unit). El sistema se basa en la presión del colector de admisión, la cual es registrada mediante la implementación física de un sensor MAP, por lo que el sistema tiene la facultad de reducir al mínimo el tiempo de diagnóstico; el sistema no utiliza variables de la calidad del aire de la ciudad, tampoco meteorológicas para el entrenamiento de la RNA, por lo que constituye un avance significativo para la predicción de emisiones de gases de escape y determinación de fallos mecánicos, resulta confiable y accesible poner en marcha este sistema en centros de servicio automotor y de revisión técnica vehicular (RTV).

\section{Métodos y materiales}

En la presente sección se desarrollan las temáticas principales que tienen que ver con la configuración experimental e instrumentación mínimamente invasiva, condiciones de adquisición de muestras, metodología para la adquisición de datos, obtención de matriz de análisis y reducción de atributos, selección de atributos para entrenamiento de la RNA y algoritmo de la red neuronal en Matlab para el diagnóstico y predicción de emisiones.

\subsection{Configuración experimental e instru- mentación mínimamente invasiva}

El estudio tiene como consideración principal evitar el despiece de elementos y sistemas del motor de un vehículo para diagnosticar sus fallas mecánicas y predecir emisiones contaminantes, por lo que se mide la depresión del motor mediante la instalación de un sensor MAP en una toma de vacío del múltiple de admisión, ubicándolo después de la mariposa de aceleración, de tal manera que la conexión no afecta el funcionamiento del MEP.
En la Tabla 1, se resumen las características del motor a prueba y en la Tabla 2, se indica la instrumentación aplicada.

Tabla 1. Características de la unidad experimental

\begin{tabular}{cc}
\hline Característica & Valor \\
\hline Modelo & Hyundai \\
Número de cilindros & 4 \\
Tren de válvulas & DOHC \\
Sistema de inyección & MFI \\
Cilindrada & $2000 \mathrm{cc}$ \\
Potencia & $175 \mathrm{CV} \mathrm{@} \mathrm{6000} \mathrm{rpm}$ \\
Torque & $168 \mathrm{~N} . \mathrm{m} \mathrm{@} \mathrm{4000} \mathrm{rpm}$ \\
Combustible & Gasolina (RON 95) \\
Índice de compresión & $10.5: 1$ \\
\hline
\end{tabular}

Tabla 2. Instrumentación aplicada

\begin{tabular}{cc}
\hline Característica & Valor \\
\hline Computadora personal & PC \\
Escáner automotor & Multiscan (Hanatech) \\
& Motor, Transmisión, \\
Conexión & ABS, Airbag. \\
& Ni DAQ-6009 \\
Diagnóstico & AI de 14 bits, a $48 \mathrm{KS} / \mathrm{s}$ \\
Tarjeta de adquisición & 2 AO de 12 bits \\
de datos & \\
Entradas & \\
Salidas & \\
Presión de admisión & MAP \\
Rango de presión & $20-117 \mathrm{KPa}$ \\
Voltaje & Piezoeléctrico \\
Tipo de sensor & \\
Analizador de gases & QROTECH / QGA 6000 \\
para vehículos & $0.0 \sim 9.99 \%$ \\
Rango de medición CO & $0.01 \%$ \\
Resolución & $0.0 \sim 9999 \mathrm{ppm}$ \\
Rango de medición HC & $1600 \mathrm{ppm}$ \\
Resolución &
\end{tabular}

La identificación respectiva de cada cilindro del motor se realiza mediante el registro de la señal del sensor de posición del árbol de levas (CMP).

En la Figura 1, se presenta la unidad experimental puesta a prueba Motor Hyundai Sonata 2.0 DOHC, un analizador de gases, una computadora personal (PC) y un escáner automotor. En la Figura 2, se muestra la conexión del sensor tipo MAP, la toma de vacío en el colector de admisión y la herramienta de adquisición de datos Ni DAQ-6009. 


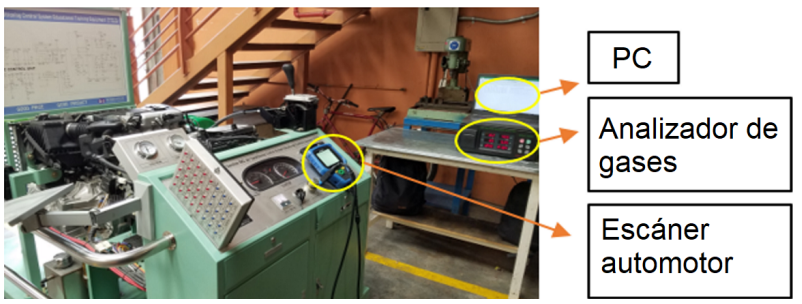

Figura 1. Instrumentación en el motor.

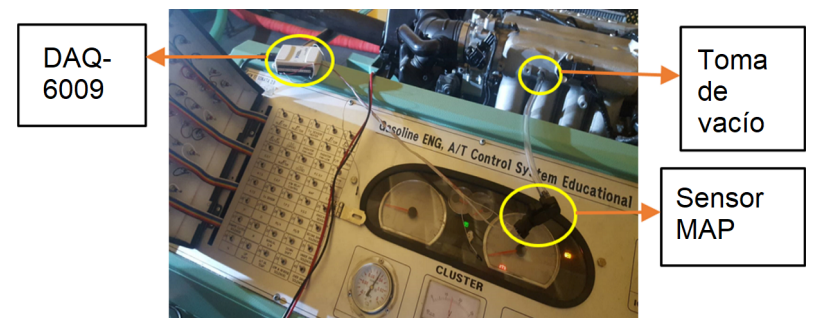

Figura 2. Conexión del sensor MAP.

\subsection{Condiciones de adquisición de muestras}

Se realiza la instalación del sensor de presión de admisión ubicándolo en el colector de admisión del MEP, consecuentemente se adquiere muestras de emisiones de $\mathrm{NO}_{x}, \mathrm{CO}, \mathrm{CO}_{2}$ y $\mathrm{HC}$ mediante el analizador de gases, la señal del sensor MAP con una tarjeta $\mathrm{Ni}$ DAQ-6009 en conjunto con el software LabVIEW se registra las muestras MAP.

Las muestras de presión y emisiones contaminantes se adquieren en ralentí aproximadamente a 850 rpm con un rango de temperatura del motor de entre 92 y $99{ }^{\circ} \mathrm{C}$, carga del motor de $35 \%$ y se utiliza el escáner automotor para corroborarlas. Sobre la base de un estudio preexperimental realizado en la investigación se determinó que la señal del sensor MAP posee picos de mayor frecuencia, de tal manera que se realiza la toma de muestras a una velocidad de 10 $\mathrm{KHz}$ en un tiempo de 5 segundos para cada una de las señales, dicha velocidad supera el criterio de Nyquist $(1.416 \mathrm{KHz})[11]$.

\subsection{Metodología para la adquisición de datos}

En la Figura 3 se presentan los elementos físicos necesarios para el correspondiente diagnóstico de fallas mecánicas y predicción de emisiones contaminantes.

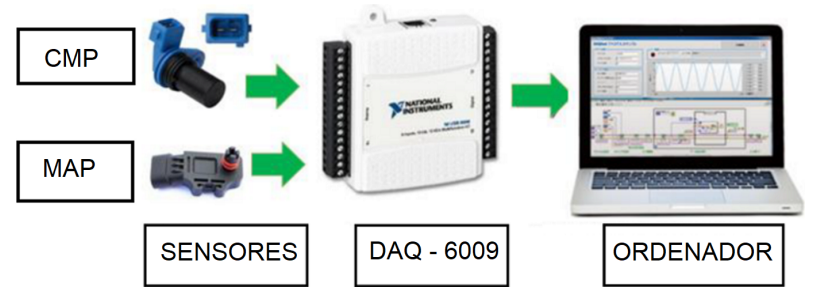

Figura 3. Elementos necesarios para la toma de datos.
Para obtener los datos de las señales de los sensores MAP y CMP se aplica el proceso representado en el flujograma de la Figura 4.

El proceso de obtención de datos se inicia con la revisión del motor en correcto funcionamiento o falla supervisada, posterior a ello se procede a inspeccionar la conexión de los sensores. Si la conexión es correcta, se graba la señal con el software Labview y se registra en un archivo de Excel, de lo contrario, se procede a verificar la conexión de los sensores [11]. Para registrar las señales se aplica el procedimiento antes descrito tanto para el caso de motor en buen funcionamiento como para el motor con falla supervisada, Figura 4 (a); 4 (b) [11]. El proceso de adquisición de datos se realiza 20 veces para cada una de las condiciones del motor.

En la Tabla 3 se indica el total de seis fallas que se generan en la unidad experimental MEP, cada una con su respectivo código de identificación; también se indica la condición del motor en óptimo funcionamiento.
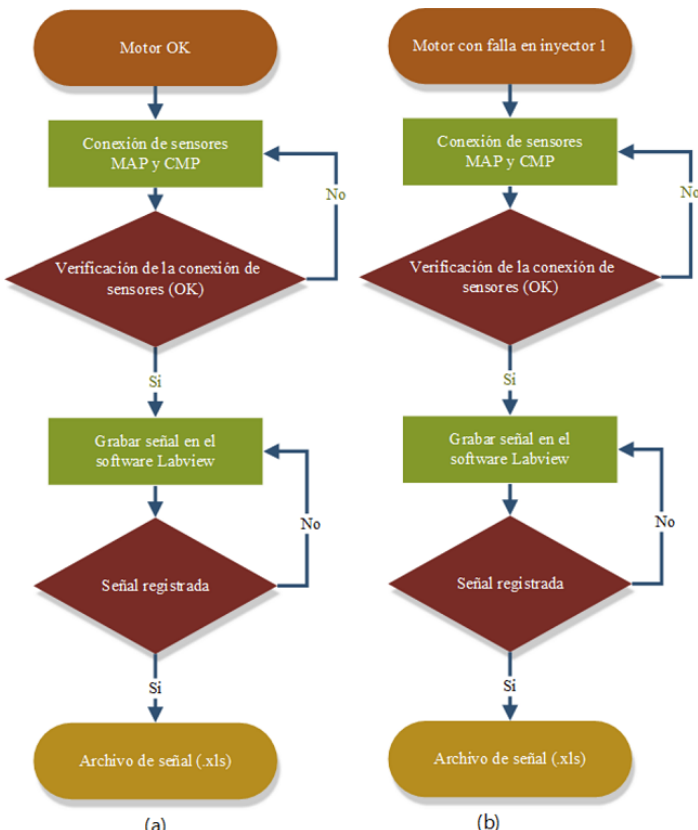

Figura 4. Flujograma de proceso para la adquisición de datos (a) motor ok, (b) motor con falla.

Tabla 3. Condiciones funcionales en la unidad experimental MEP

\begin{tabular}{ccc}
\hline N. & $\begin{array}{c}\text { Tipo de condición } \\
\text { mecánica }\end{array}$ & $\begin{array}{c}\text { Código de } \\
\text { identificación }\end{array}$ \\
\hline 1 & Motor en óptimo & 100 \\
2 & funcionamiento & 200 \\
3 & Falla inyector 1 & 300 \\
4 & Falla inyector 2 & 400 \\
5 & Falla inyector 3 & 500 \\
6 & Falla inyector 4 & 1000 \\
7 & Falla en bobina 1-4 & 1100 \\
\hline
\end{tabular}




\subsection{Obtención de matriz de análisis y reduc- ción de atributos}

Se realiza un corte completo en la señal del sensor MAP que corresponde a un ciclo del motor $\left(720^{\circ} \pm 180^{\circ}\right)$, tomando en cuenta el reglaje de distribución de adelanto a la apertura de admisión (AAA) y retraso de cierre de admisión (RCA), correspondiente a cada uno de los cilindros [11]. Se desarrolla un ventaneo de la señal del sensor tipo MAP para cada cilindro, la cual se observa en la Figura 5.

Una vez realizada la toma de señales temporales se procede a desarrollar un algoritmo en el software Matlab para la correspondiente lectura y obtención de matriz general con 18 atributos, que son: media geométrica, máximo, mínimo, mediana, covarianza, varianza, desviación estándar, moda, factor de curtosis, coeficiente de asimetría, energía potencia, área bajo la curva, entropía, coeficiente de variación, rango, raíz media cuadrática y factor de cresta [11].
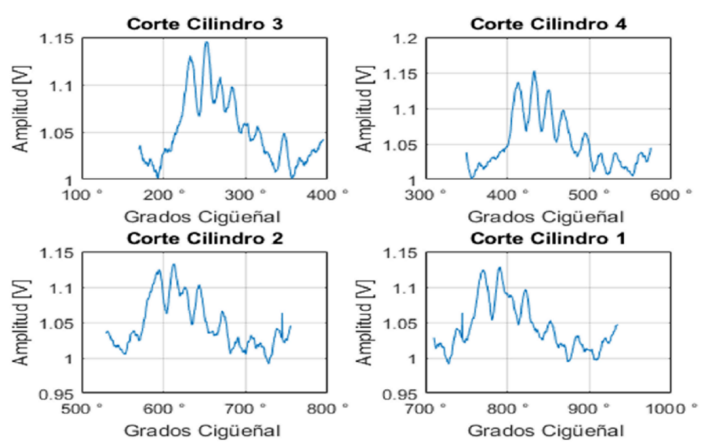

Figura 5. Ventaneo de la señal del sensor tipo MAP por cada cilindro.

Para la selección y reducción del número de atributos se analiza la matriz general a través de 3 métodos estadísticos: ANOVA, matriz de correlación y Random Forest.

La aplicación del método estadístico ANOVA de un solo factor, permite determinar los mejores atributos que ingresan a la matriz general, mediante el análisis de los 18 atributos, tomando en consideración en el método el mayor valor de $\mathrm{R}^{2}$ puesto que los valores cercanos al $100 \%$ indican que existe un correcto ajuste de los datos al modelo, es decir, se determina la variación entre los atributos. Además, en el método se consideran los valores de p próximos a 0 que determinan si los atributos son estadísticamente significativos [11].

Respecto a la matriz de correlación, se descartaron los atributos que contienen coeficientes próximos a -1 o 1, dado que con los mismos existe una relación fuerte entre las variables, ya sea negativa o positiva, respectivamente. En efecto, se seleccionaron los atributos con coeficientes próximos a cero, puesto que con estos atributos no existe una correlación fuerte entre las variables [11].
En cuanto al método de Randon Forest, permite obtener la estimación de importancia de atributos con uso de los métodos de Curvature test, Standard CART e Interaction test. Posterior a ello, se aplicó el análisis de Pareto para seleccionar los atributos de mayor prioridad, considerando solo el primer $95 \%$ de la distribución acumulada [11].

\subsection{Selección de atributos para entrenamiento de la RNA}

Para seleccionar los atributos que serán considerados en la entrada de la red neuronal se realizó un análisis de coincidencia de la matriz general de la cual se seleccionaron los atributos que más se repiten entre los resultados de cada método estadístico aplicado [11]. Los atributos que más se repiten se muestran en la Tabla 4.

Tabla 4. Atributos utilizados para entrenar la red neuronal artificial

\begin{tabular}{cc}
\hline $\begin{array}{c}\text { Atributos } \\
\text { estadísticos }\end{array}$ & $\begin{array}{c}\text { Número de } \\
\text { repeticiones }\end{array}$ \\
\hline Área $\left(\mathrm{v}^{2}\right)$ & 5 \\
Energía $(\mathrm{J})$ & 5 \\
Entropía $(\mathrm{J})$ & 4 \\
Máximo $(\mathrm{V})$ & 4 \\
Media $(\mathrm{V})$ & 5 \\
Mínimo $(\mathrm{V})$ & 5 \\
Potencia $(\mathrm{mW})$ & 5 \\
RMS $(\mathrm{V})$ & 5 \\
\hline
\end{tabular}

\subsection{Algoritmo de la red neuronal en Matlab para el diagnóstico y predicción de emi- siones}

Mediante el uso de la plataforma de RNA del software Matlab se realizaron diferentes configuraciones para obtener dos redes neuronales para la emisión contaminante $\mathrm{CO}$ y $\mathrm{HC}$, respectivamente, con un mínimo error de clasificación.

En la Figura 6 se presenta el flujograma para la creación de las respectivas redes neuronales artificiales de $\mathrm{CO}$ y $\mathrm{HC}$.

El algoritmo inicia con la lectura de la matriz de entrada y respuesta respectiva para la RNA. Luego se normaliza el vector de entrada y la respuesta con el valor máximo de cada matriz con el objetivo de optimizar la creación de la RNA. Una vez normalizada la matriz de atributos se procedió a la creación de la RNA [11].

Las redes neuronales se establecen de acuerdo con las características indicadas en la Tabla 5. 


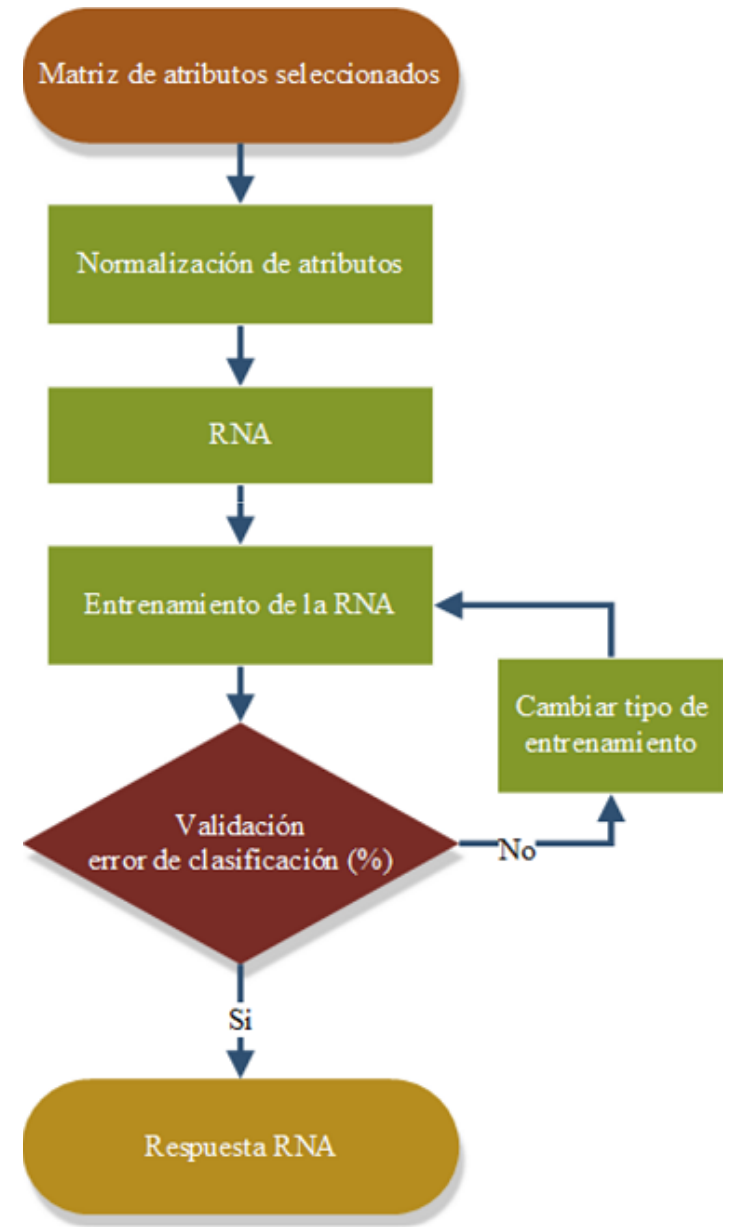

Figura 6. Flujograma de creación de RNA.

Tabla 5. Atributos utilizados para entrenar la red neuronal artificial

\begin{tabular}{cc}
\hline Característica & Tipo \\
\hline Modelo & Backpropagation \\
Tipología & 4 capas Feedforward \\
On line/Off line & Off \\
Aprendizaje & No supervisado \\
Regla de aprendizaje & Corrección de error \\
Asociación & Heteroasociación \\
Salida & Análoga \\
\hline
\end{tabular}

En la Figura 7 se observan los parámetros de la creación de la RNA de tipo feed-forward backpropagation para la predicción de la emisión contaminante CO.

En la Figura 8 se observan los parámetros de la creación de la RNA de tipo feed-forward backpropagation para la predicción de la emisión contaminante $\mathrm{HC}$.

Una vez creadas las redes, se procedió a su entrenamiento y para ello se consideraron los parámetros como: tipo de algoritmo, número de épocas y el error máximo.
A continuación, se presentan los pasos y fórmulas a utilizar para entrenar la red neuronal:

1. Los pesos de la red neural se inicializan con valores pequeños aleatorios.

2. Se ingresa un patrón de entrada a la red con las diferentes condiciones del motor Xp (Xp1, Xp2, ... Xpn) y se especifica la salida deseada de la red como Ym que sería el valor de emisiones.

3. Se calcula la salida actual de la red.

La arquitectura de la red se muestra en la Figura 9 , donde el subíndice $p$ indica el p-ésimo vector de entrenamiento, $\mathrm{j}$ el número de neurona oculta y el índice i varía desde 1 hasta el número de entradas de la capa de entrada.

Con las redes previamente entrenadas se verificó el error de clasificación, respectivamente, en la red de $\mathrm{CO}$ y red $\mathrm{HC}$, si el error es mayor al $5 \%$, se procede a cambiar los parámetros para disminuir el error de clasificación.

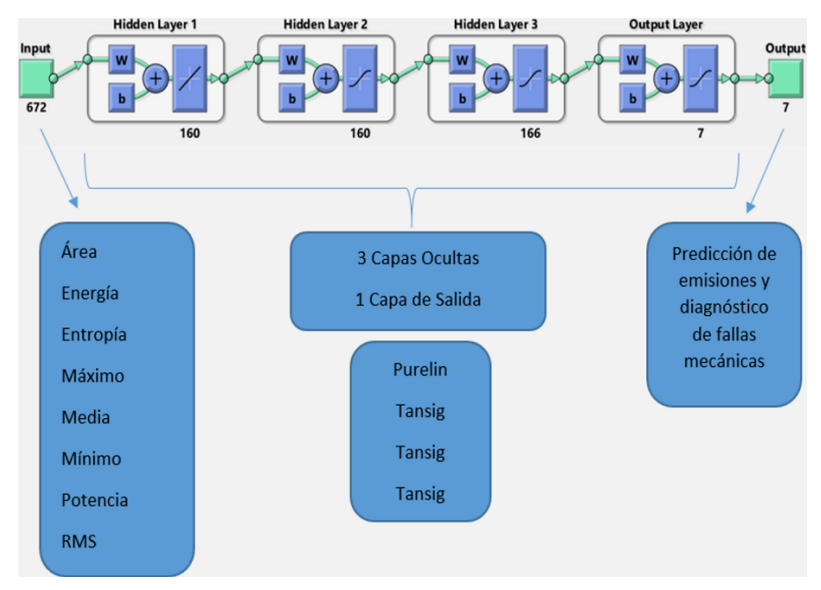

Figura 7. Estructura de la red neuronal CO.

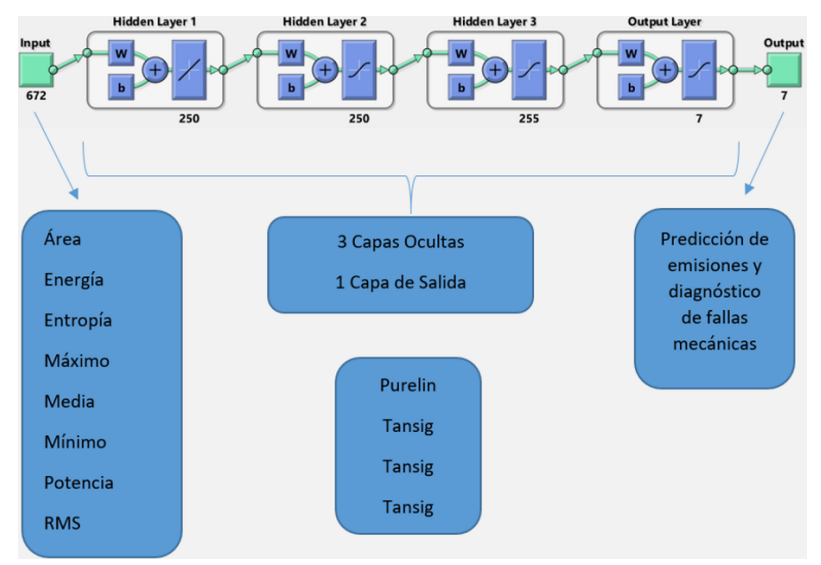

Figura 8. Estructura de la red neuronal HC. 


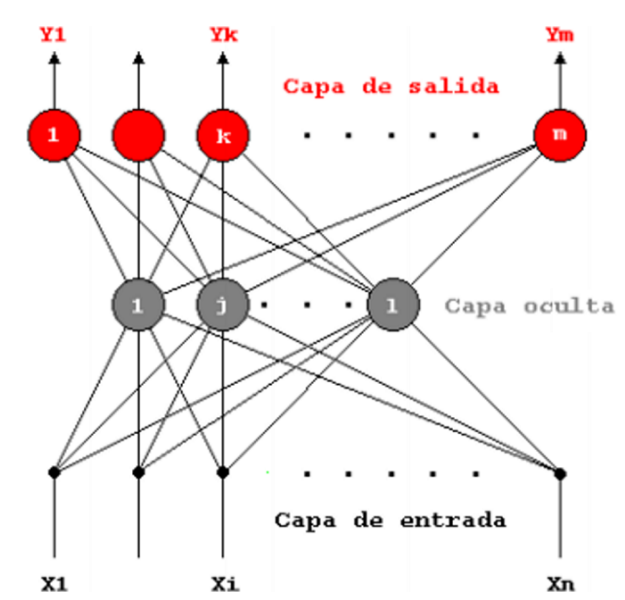

Figura 9. Arquitectura de la red feed-forward.

La red neuronal de CO utiliza la función de entrenamiento trainscg (Scaled Conjugate Gradient) para el sistema de predicción de emisiones y diagnóstico de fallas mecánicas, el cual presentó un error de $5.4061 \mathrm{e}^{-9}$.

Así mismo la red neuronal de la emisión $\mathrm{HC}$ utiliza la función de entrenamiento trainscg para la predicción de emisiones y diagnóstico de fallas mecánicas, la cual presentó un error de $9.7587 \mathrm{e}^{-5}$.

En la Figura 10 y Figura 11 se presentan los resultados del coeficiente de correlación de Pearson $\mathrm{R}$ de la red neuronal de $\mathrm{CO}$ y $\mathrm{HC}$, respectivamente, la cual es facilitada por el código de entrenamiento red.trainFcn $={ }^{\prime}$ trainscg ${ }^{\prime}$ del software Matlab.
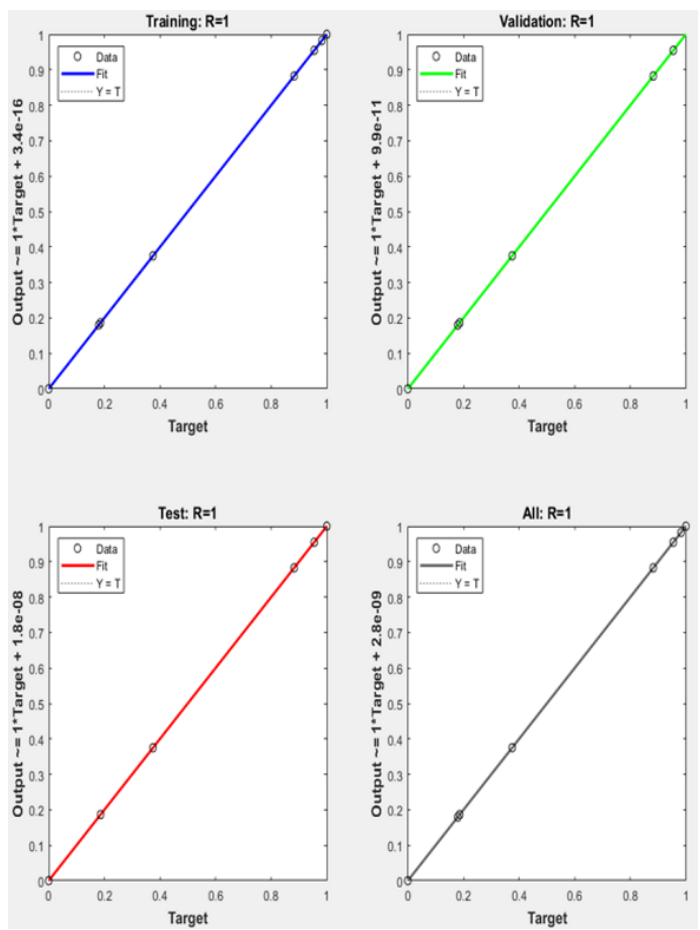

Figura 10. Correlación entre los valores esperados y pronosticados por la red neuronal $\mathrm{CO}$.
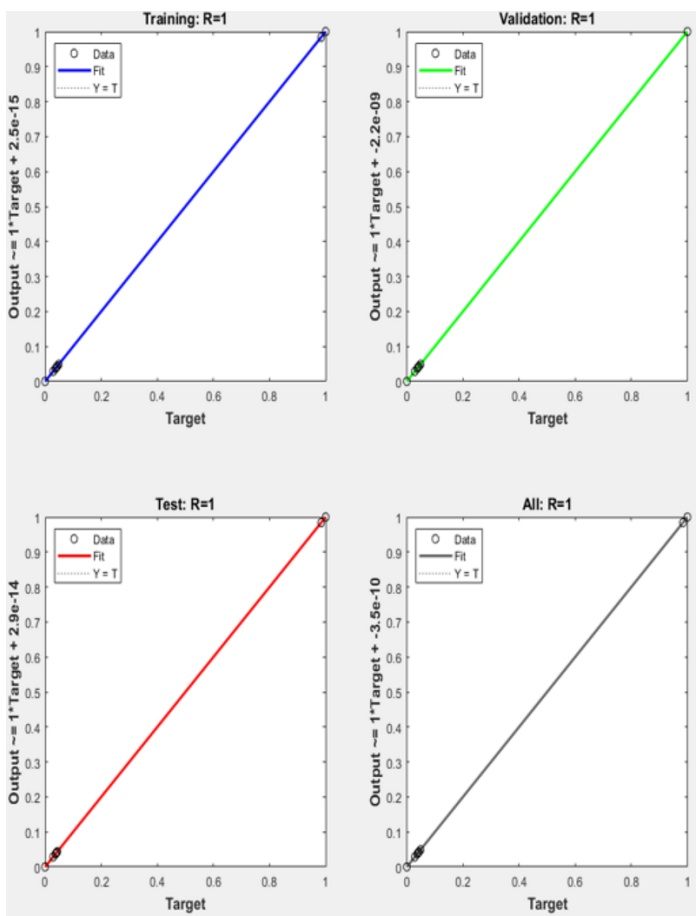

Figura 11. Correlación entre los valores esperados y pronosticados por la red neuronal HC.

Las líneas indican los valores esperados y los círculos negros representan los valores pronosticados por la RNA. La predicción determinada por la red neuronal es eficiente y se corrobora con un buen desempeño, dado que se obtiene un índice global de 1 en entrenamiento, validación y prueba lo que indica una relación lineal fuerte positiva entre las condiciones reales del MEP y los resultados proporcionados por la red neuronal [11].

En las Figuras 12 y 13 se muestra la comparación entre la respuesta de la red neuronal con el resultado esperado, respectivamente de la red $\mathrm{CO}$ y red $\mathrm{HC}$; se observan las siete condiciones mecánicas reales del motor identificadas por la red neuronal.

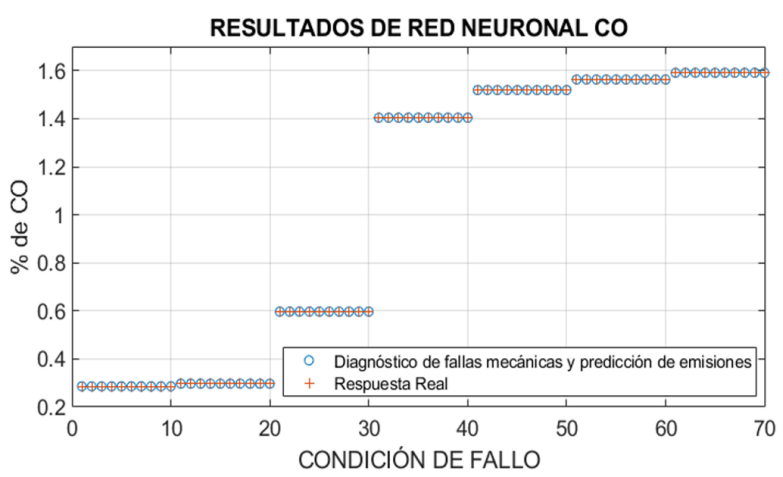

Figura 12. Red neuronal CO con porcentaje de error de $5.4061 \mathrm{e}^{-9}$, con función de entrenamiento "trainscg". 


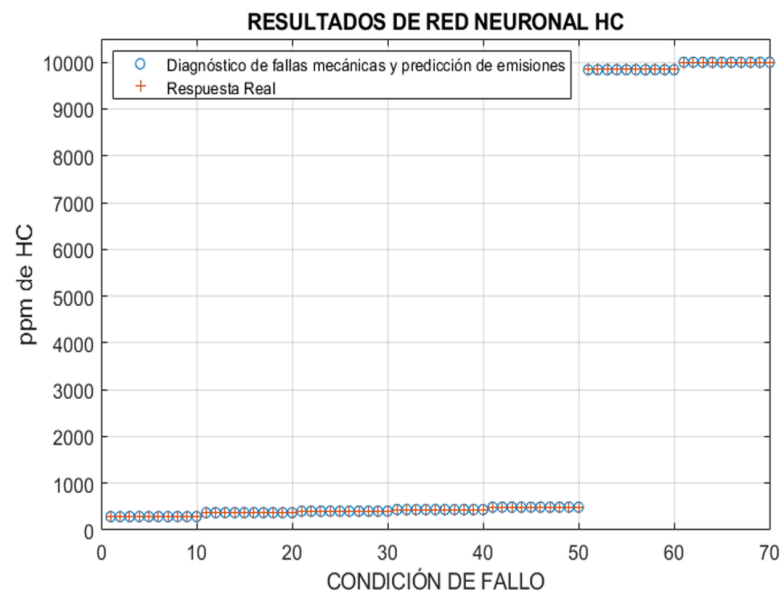

Figura 13. Red neuronal HC con porcentaje de error de $9.7587 \mathrm{e}^{-5}$, con función de entrenamiento "trainscg".

\section{Resultados y discusión}

A fin de comparar el correcto funcionamiento del sistema de predicción de emisiones y diagnóstico de fallas mecánicas se procede a realizar varias pruebas bajo diversas condiciones de funcionamiento.

En este apartado se presentan dos condiciones de falla en específico: el inyector $2(300)$ y el fallo en bobina 1-4 (1000).

En la Figura 14 se muestran los resultados de los valores obtenidos por la red neuronal CO para los estados de funcionamiento cuando el inyector 2 se encuentra con falla.

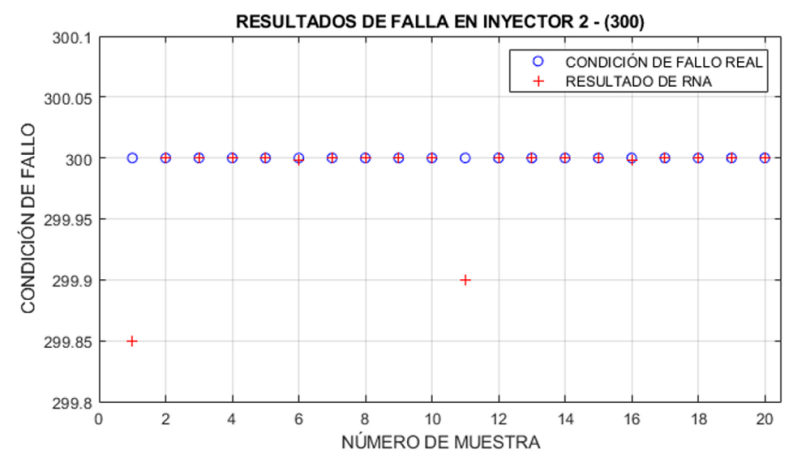

Figura 14. Resultado de condición de funcionamiento de inyector 2 .

En la Figura 15 se observan los resultados de los valores obtenidos por la RNA de HC para condiciones de funcionamiento cuando la bobina 1-4 se encuentra con falla.

Obtenidos los resultados de las condiciones funcionales del motor de encendido provocado, se puede resaltar que la diferencia entre las respuestas tanto de condición real y respuesta de red neuronal de $\mathrm{CO}$ y $\mathrm{HC}$ tienen un valor próximo a cero. Por lo tanto, la aplicación del sistema de diagnóstico de fallas mecánicas y predicción de emisiones contaminantes es capaz de detectar la condición funcional de falla mecánica y predicción de emisión contaminante.

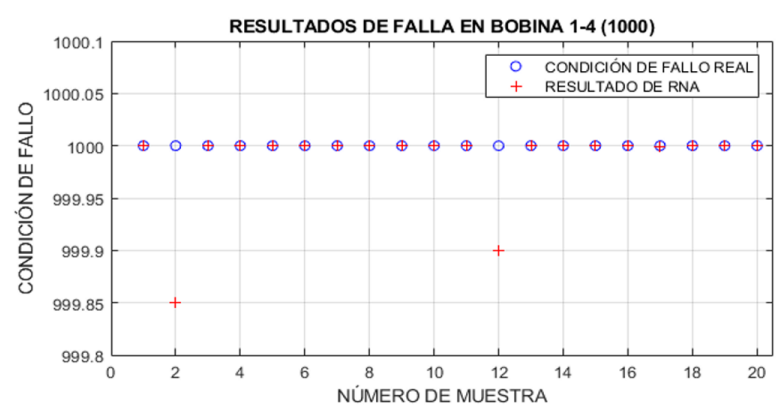

Figura 15. Resultado de condición de funcionamiento de bobina de ignición de alta tensión 1-4.

En efecto en la Figura 16 se observa que al agrupar los datos de condición real del motor y respuesta obtenida de la RNA de CO y en la Figura 17 la del HC, mediante el método estadístico de Tukey con un índice de confianza del $95 \%$, se determina que las medias son equivalentes y no existe una diferencia estadísticamente significativa, ya que las medias de cada una de las respuestas coinciden en un valor próximo a cero.

Además, las Figuras 18 y 19 de intervalos de la RNA CO y HC, respectivamente, indican que no existe diferencia entre los promedios de las pruebas en las diferentes condiciones funcionales del MEP.

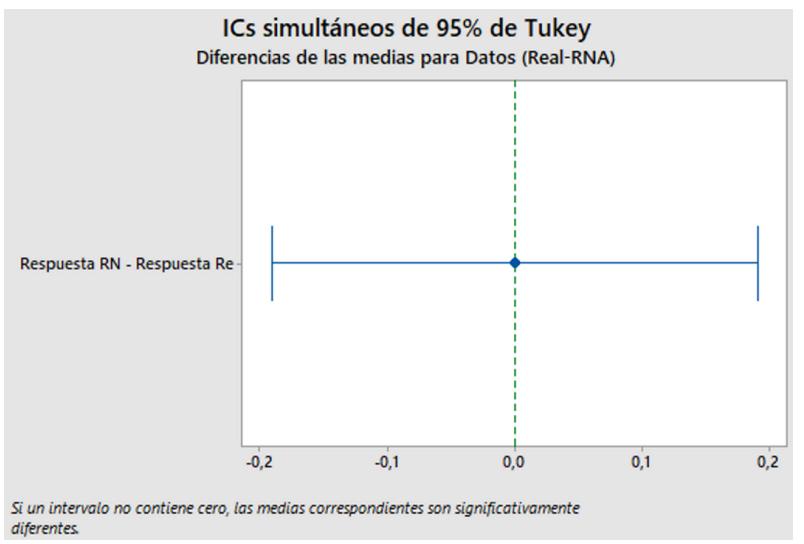

Figura 16. Gráfica de diferencias de las medias para datos de respuesta real vs. red neuronal CO. 


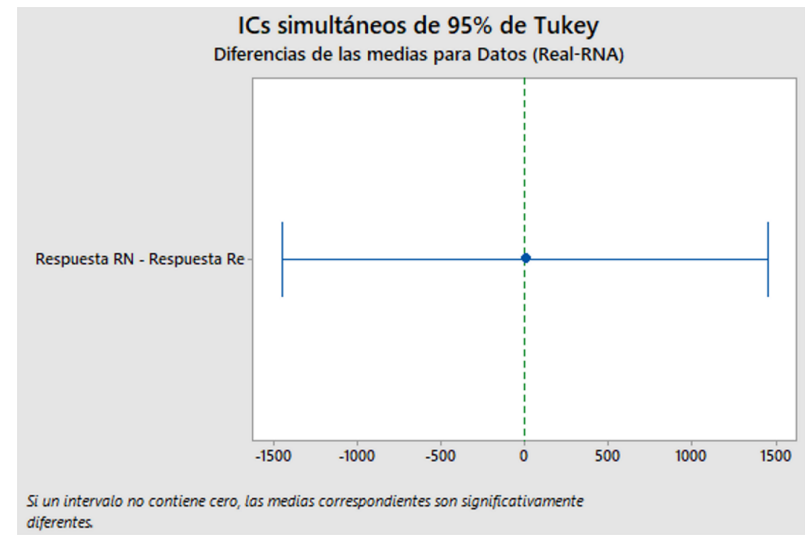

Figura 17. Gráfica de diferencias de las medias para datos de respuesta real vs. red neuronal HC.

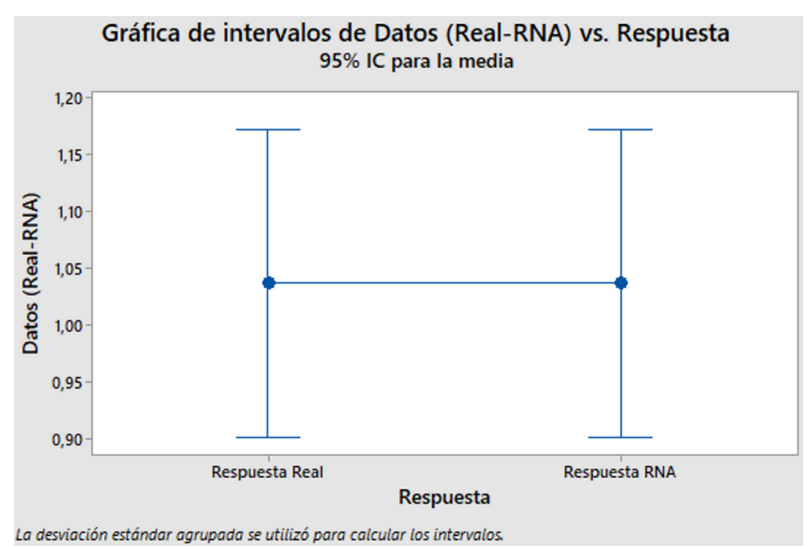

Figura 18. Gráfica de intervalos de datos de respuesta real vs. red neuronal CO.

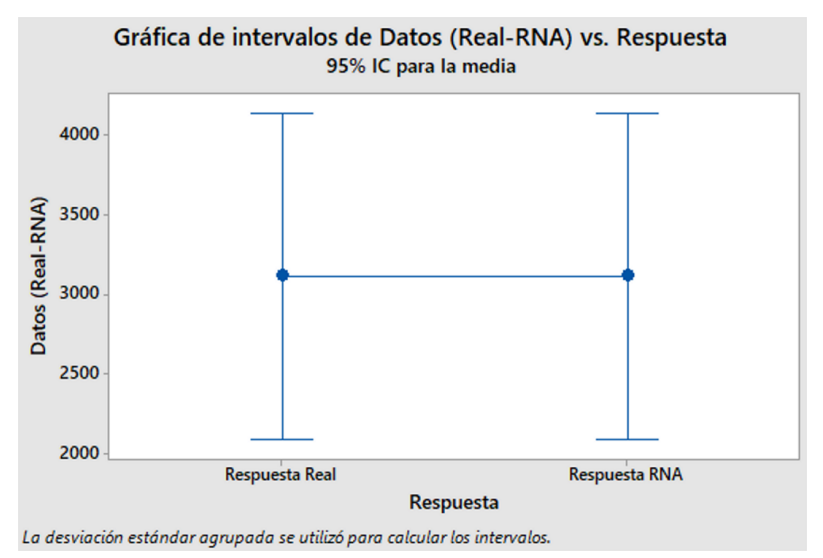

Figura 19. Gráfica de intervalos de datos de respuesta real vs. red neuronal $\mathrm{HC}$.

Asi mismo, en las Figuras 20 y 21 se corrobora que existe una relación entre la respuesta real y de la red neuronal ya que comparten la misma letra de agrupación (A) y el valor $p$ ( $p$-valúe) es igual a 1. Dando como resultado un valor de confiabilidad aproximadamente del 100,00\%, siendo este valor aceptable para temas de diagnóstico de fallas mecánicas y predicción de emisiones contaminantes de motores de combustión interna de encendido provocado.

$$
\begin{aligned}
& \text { Análisis de Varianza } \\
& \begin{array}{lrrrrr}
\text { Fuente } & \text { GL } & \text { SC Ajust. } & \text { MC Ajust. } & \text { Valor } F & \text { Valor } p \\
\hline \text { Respuesta } & 1 & 0,0000 & 0,000000 & 0,00 & 1,000 \\
\text { Error } & 138 & 45,1161 & 0,326928 & & \\
\text { Total } & 139 & 45,1161 & & &
\end{array}
\end{aligned}
$$

\section{Comparaciones en parejas de Tukey}

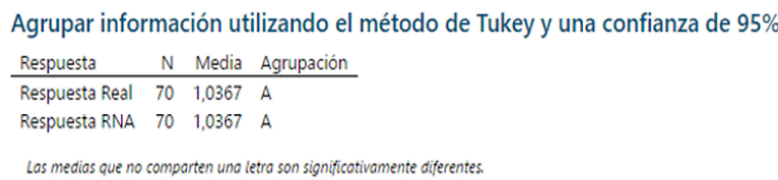

Figura 20. Resultados del análisis de varianza y comparaciones en parejas de Tukey de la RNA de CO.

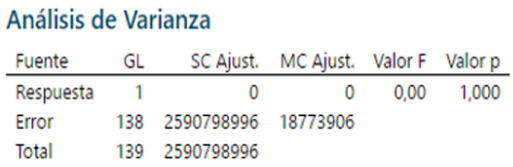

Comparaciones en parejas de Tukey

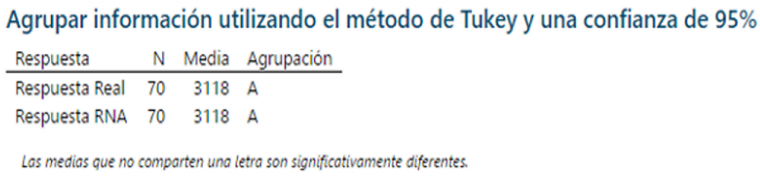

Figura 21. Resultados del análisis de varianza y comparaciones en parejas de Tukey de la RNA de HC.

\section{Conclusiones}

Los modelos de redes neuronales para el diagnóstico y predicción de emisiones contaminantes tanto de $\mathrm{CO}$ y HC desarrollados posee un error de clasificación de $5.4061 \mathrm{e}^{-9}$ y $9.7587 \mathrm{e}^{-5}$, respectivamente.

La función de entrenamiento trainscg, permite la identificación precisa de los diferentes tipos de condiciones mecánicas del MEP y predicción de emisiones, por lo que constituye una alternativa viable para ser integrada en un sistema de diagnóstico como escáner automotor o analizador de gases de vehículos a gasolina, debido a la rapidez computacional que ofrecen las redes neuronales artificiales.

Mediante el desarrollo del análisis de varianza de un solo factor, se obtuvo un valor de $p(p$-valúe $=1,00)$ demostrando que la respuesta real de clasificación de fallas mecánicas y predicción de emisiones es equivalente al resultado obtenido mediante las redes neuronales desarrolladas, de manera que con este valor se demuestra que no existe una diferencia estadísticamente significativa. 
En este trabajo se muestra que la aplicación de redes neuronales (feed-forward backpropagation) es válida para la detección de condiciones de fallas mecánicas como también para la predicción de emisiones contaminantes de vehículos a gasolina, además, la técnica de diagnóstico aplicada presenta la ventaja de evitar el desensamble de elementos y sistemas del motor con una técnica mínimamente invasiva fiable y de gran precisión.

Los resultados muestran que las redes neuronales (feed-forward backpropagation) con 160 o 250 neuronas ocultas y entrenando con la función trainscg (Scaled Conjugate Gradient), puede lograrse un error promedio $4.87962 \mathrm{e}^{-5}$, lo que demuestra que las emisiones de vehículos a gasolina se pueden predecir con alta precisión.

\section{Referencias}

[1] F. Ballester, "Contaminación atmosférica, cambio climático y salud," Revista Española de Salud Pública, vol. 79, pp. 159-175, 04 2005. [Online]. Available: https://bit.ly/2piHMQZ

[2] A. Restrepo, S. Izquierdo, and R. López, "Estimación de factores que inciden sobre la contaminación ambiental generada por fuentes móviles en Pereira," Scientia et technica, vol. 1, no. 37, pp. 267-272, 2007. [Online]. Available: http://dx.doi.org/10.22517/23447214.4053

[3] X. Li, D. Sun, G. Lu, J. Krabicka, and Y. Yan, "Prediction of nox emissions throughflame radical imaging and neural network based soft computing," in 2012 IEEE International Conference on Imaging Systems and Techniques Proceedings, July 2012, pp. 502-505. [Online]. Available: https://doi.org/10.1109/IST.2012.6295594

[4] M. Cortina, "Aplicación de técnicas de inteligencia artificial a la predicción de contaminantes atmosféricos," Ph.D. dissertation, 2012.

[5] J. D. Martínez-Morales, E. R. Palacios-Hernández, and G. A. Velázquez-Carrillo, "Modeling and multi-objective optimization of a gasoline engine using neural networks and evolutionary algorithms," Journal of Zhejiang University SCIENCE $A$, vol. 14, no. 9, pp. 657-670, Sep 2013. [Online]. Available: https://doi.org/10.1631/jzus.A1300010

[6] T. Fontes, L. M. Silva, S. R. Pereira, and M. C. Coelho, "Application of artificial neural networks to predict the impact of traffic emissions on human health," in Progress in Artificial Intelligence, L. Correia, L. P. Reis, and J. Cascalho, Eds. Berlin, Heidelberg: Springer Berlin Heidelberg, 2013, pp. 21-29. [Online]. Available: https://doi.org/10.1007/978-3-642-40669-0_3

[7] J. D. Martínez-Morales, E. R. Palacios-Hernández, and G. A. Velázquez-Carrillo, "Artificial neural network based on genetic algorithm for emissions prediction of a SI gasoline engine," Journal of Mechanical Science and Technology, vol. 28, no. 6, pp. 2417-2427, Jun 2014. [Online]. Available: https://doi.org/10.1007/s12206-014-0535-2

[8] T. López Ortíz and A. Pacheco González, "Efectos de la contaminación atmosférica en la salud de las personas en la ciudad de Cuenca," 2015. [Online]. Available: https://bit.ly/32M8jnz

[9] J. Guadaluoe Almeida, "Modelación de emisiones contaminantes de fuentes móviles terrestres en Quito, Ecuador," 2016. [Online]. Available: https://bit.ly/2qdvtG8

[10] P. León Bacuilima and C. Piña Orellana, "Predicción de emisiones contaminantes de gases de escape a través de la presión media efectiva empleando redes neuronales en motores de encendido provocado, Cuenca," 2018. [Online]. Available: https://bit.ly/2q2oTlT

[11] W. Contreras, J. Maldonado, and R. León, "Aplicación de una red neuronal feed-forward backpropagation para el diagnóstico de fallas mecánicas en motores de encendido provocado," INGENIUS, 2019. [Online]. Available: https://doi.org/10.17163/ings.n21.2019.03 